\title{
Antegrade Brain Perfusion for Pulmonary Thromboendarterectomy
}

\author{
Jean Francois Morin, Andrew Hirsch, Senthuran Tharmalingam \\ Jewish General Hospital Division of Respiratory Medicine, McGill University, Montreal, Canada \\ E-mail: \{jmorin, ahirsch\}@jgh.mcgill.ca, senthuran_t@hotmail.com \\ Received September 28, 2011; revised October 30, 2011; accepted November 11, 2011
}

\begin{abstract}
Objective: The gold standard procedure for pulmonary thromboendarterectomy is median sternotomy, cardiopulmonary bypass, profound hypothermia $\left(18^{\circ} \mathrm{C}\right)$ and circulatory arrest. We propose a modified technique to improve the quality of care in this patient population, based on an intervention previously used in aortic surgery. Method: In our modified technique, we cannulated the right axillary artery to allow antegrade brain perfusion while on circulatory arrest. In this retrospective study, we have reviewed the data relating to the first 7 patients on whom we performed the modified technique and have made comparison with a group of 7 case-matched individuals who underwent the standard technique (control group). Results: The modified technique allowed for use of moderate hypothermia $\left(25^{\circ} \mathrm{C}-28^{\circ} \mathrm{C}\right)$. Patients in both groups woke up without neurologic complications. A trend towards, but non-significant reduction in duration of surgery from 303 $( \pm 42)$ to $279( \pm 44)$, duration of postoperative inotropic support from $2.7 \pm 3.4$ days to $1.7 \pm 2.0$ days, as well as postoperative mechanical ventilation time from $4.87( \pm 3.7)$ to $2( \pm 2.7)$ days were seen in the control and modified groups respectively. All patients in the modified group woke up on post-operative day 0 , whereas most patients in the control group awoke on postoperative day 1 . No significant differences were noted in the reduction in preoperative to postoperative systolic pulmonary artery pressure, post-operative length of stay in the intensive care unit and length of stay in the hospital among the two groups. Conclusions: The antegrade brain perfusion via the right axillary artery allows for good brain protection, while maintaining a bloodless field in the arterial pulmonary tree. All our patients awoke without any neurologic deficits. In the future, by using an even milder level of cooling, we may be able to significantly reduce the duration of surgery and improve the recovery of our patients.
\end{abstract}

Keywords: Cerebral Perfusion, Pulmonary Thromboendarterectomy

\section{Introduction}

Pulmonary thromboendarterectomy (PTE) offers a surgical cure for pulmonary hypertension due to thromboembolic disease [1]. The standard technique for thromboendarterectomy, as developed at the University of San Diego (UCSD), involves median sternotomy, cardiopulmonary bypass and intermittent periods of deep hypothermic circulatory arrest (DHCA) to $18^{\circ} \mathrm{C}$ [1]. Using this method, the UCSD group reported $17 \%$ operative mortality in their first 200 cases but have decreased it to below $5 \%$ in the last 500 , suggesting a learning curve associated with the procedure [2]. Another group, Thomson et al., reported a $15 \%$ mortality in their total cohort of 150 patients, but only $4 \%$ in the last 25 patients (Thom- son).

It has also been reported that the use of DHCA is associated with longer duration of surgery, adverse postoperative neurologic outcomes due to derangements in cellular metabolism [3] and coagulation disorders promoting haemorrhage [4]. We also hypothesize that DHCA is associated with increased postoperative wake up time, mechanical ventilation time and longer use of postoperative inotropic support.

To enable an increase in circulatory arrest time and alleviate the need for deep hypothermia, there have been attempts by several groups to use antegrade cerebral brain perfusion to improve operative morbidity in PTE $[2,5,6]$. The concept of antegrade cerebral brain perfusion had previously been successfully implemented in 
aortic arch surgery [7].

In a retrospective study, we present our results in 7 patients who underwent selective antegrade brain perfusion at moderate hypothermia.

\section{Patients and Methods}

Prior to 2008, all patients at our center underwent the standard technique. Since 2008, antegrade cerebral perfusion has been used in PTE. In this retrospective study, we have reviewed the data from the first seven patients who underwent the modified procedure. We then made comparison with the last seven case-matched patients who underwent the standard technique, as developed at the UCSD (control group) [1].

The preoperative characteristics of the patients are described in Table 1.

The indication for operation was established after clinical assessment and hemodynamic parameters (on the basis of pulmonary function tests, echocardiogram, and right heart catheterization). An inferior vena cava (IVC) catheter was placed in all patients preoperatively.

\subsection{Surgical Technique}

General anaesthesia was induced. Following this, a transverse incision is made below the right clavicle, until the right axillary artery is identified. An $8 \mathrm{~mm}$ Hemashield sidegraft is sewed to the right axillary artery [8]. One end of a cannula is inserted into the sidegraft, while the other is connected to the cardiopulmonary bypass machine.

Following this, a median sternotomy is performed and the pericardium is opened. Systemic heparinization is administered to achieve an activated clotting time of greater than $500 \mathrm{~s}$. The innominate artery and left com- mon carotid arteries are exposed. The superior vena cava (SVC) and inferior vena cava (IVC) are cannulated for cardiopulmonary bypass. After the institution of bypass, the body is cooled to moderate hypothermic temperatures of $25^{\circ} \mathrm{C}-28^{\circ} \mathrm{C}$. Once the heart fibrillates, the aorta is cross clamped and cardioplegia is delivered through a cannula inserted into the ascending aorta. The cardiopulmonary bypass pump is stopped. The origin of the innominate artery and left carotid arteries are clamped, so as to prevent backflow. Then, antegrade perfusion of the brain via the right axillary artery is begun, maintaining a perfusion pressure of $50 \mathrm{mmHg}$.

Endarterectomy is begun with a longitudinal incision of the right pulmonary artery. The endarterectomy plane is extended distally as far as needed into the affected segmental or subsegmental vessles. After completion, the right arteriotomy is then closed, and the innominate and left carotid arteries are unclamped and the CPB pump is restarted for 10 - 15 minutes. After which, the pump is once again stopped, the innominate and left carotid arteries are clamped, antegrade cerebral perfusion is restarted and endarterectomy of the left side is begun in a similar fashion.

Following bilateral endarterectomy, the CPB machine is restarted and the patient is re-warmed to a temperature of $36.5^{\circ} \mathrm{C}$.

\subsection{Statistical Analysis}

The software, StatView, was used for statistical analysis. Continuous data are expressed as mean with standard deviation or median with range, while categorical data are expressed as total number of occurrences. Where applicable, parameters were analyzed using the Student $t$-test.

Table 1. Preoperative characteristics.

\begin{tabular}{|c|c|c|c|}
\hline Preoperative parameters & Control & Modified & $p$ Value \\
\hline Age $(y$, mean $\pm \mathrm{SD})$ & $47 \pm 11$ & $56 \pm 7$ & 0.09 \\
\hline Female:Male & $5: 2$ & $4: 3$ & \\
\hline $\mathrm{BMI}\left(\mathrm{kg} / \mathrm{m}^{\wedge} 2\right.$, mean $\left.\pm \mathrm{SD}\right)$ & $28 \pm 9$ & $28 \pm 7$ & 0.97 \\
\hline $\begin{array}{c}\text { NYHA class } \\
\text { I } \\
\text { II } \\
\text { III } \\
\text { IV }\end{array}$ & $\begin{array}{l}0 \\
0 \\
7 \\
0\end{array}$ & $\begin{array}{l}0 \\
1 \\
5 \\
1\end{array}$ & \\
\hline Previous DVT or PE & 6 & 7 & \\
\hline Mean pre-operative sPAP & $89 \pm 26$ & $77 \pm 8$ & 0.25 \\
\hline Coagulation problem (APA, LPA) & 2 & 0 & \\
\hline
\end{tabular}




\section{Results}

As expected, all patients, in both groups, showed a significant reduction in systolic pulmonary artery pressure (sPAP). The average difference in preoperative to postoperative sPAP were $28.6 \pm 12.9$ to $37 \pm 12.2 \mathrm{mmHg}$ in the modified and control groups, respectively $(p=0.23)$.

The mean duration of total circulatory arrest times were $56.1 \pm 12.5$ vs. $48.6 \pm 15.0$ minutes in the modified and control groups, respectively $(p=0.33)$. The right sided circulatory arrest times were $32 \pm 10.9$ vs. $25.6 \pm$ 12.8 minutes in the modified and control groups, respectively $(p=0.33)$. The left sided circulatory arrest times were $24.1 \pm 3.6$ vs. $23 \pm 6.4$ minutes in the modified and control groups, respectively $(p=0.69)$.

The total duration of surgery was $279 \pm 44$ vs. $303 \pm$ 42 minutes in the modified and control groups, respectively $(p=0.33)$.

Postoperative $8 \mathrm{~h}$ blood loss was measured in all patients using the mediastinal chest sump tube. The measured blood loss was $824 \pm 698$ vs. $576 \pm 448 \mathrm{ml}$ in the modified and control groups, respectively $(p=0.44)$.

All patients in the modified group awoke postoperatively on the day of surgery, however only one patient in the control group awoke on the day of surgery, while the others awoke on postoperative day 1 . The mean mechanical ventilation time was $2 \pm 2.7$ vs. $4.9 \pm 3.7$ days for the modified and control groups, respectively $(p=0.13)$. The modified group required a mean of $1.7 \pm 2.0$ days of postoperative inotropic support, as opposed to $2.7 \pm 3.4$ days required for the control group $(p=0.50)$. The median length of ICU stay was 6 days (range of 2 - 18) for the modified group and 8 days (range of 2 - 31) for the control group. The median length of hospital stay was 23 days (range of 10 - 39) for the modified group as opposed to 16 days (range of 7 - 46) for the control group.

All patients survived the operation. There were no permanent neurologic complications seen in the control nor modified groups. One patient developed transient postoperative confusion in the control group. Other postoperative complications between the two groups are recorded in Table 2 .

\section{Discussion}

Although the series is small, our results confirm that PTE with moderate hypothermia and antegrade cerebral perfusion is a safe alternative to the standard technique. The use of right axillary perfusion allows for cerebral circulation while performing the endarterectomy. There were no permanent or transient neurologic deficits reported in our modified group.

To our knowledge, this is the first report of PTE with right axillary antegrade cerebral perfusion with moderate
Table 2. Postoperative complications.

\begin{tabular}{ccccc}
\hline \multirow{2}{*}{ Postoperative complications } & \multicolumn{2}{c}{ Control } & \multicolumn{2}{c}{ Modified } \\
\cline { 2 - 5 } & No. & \% & No. & $\%$ \\
\hline Death & 0 & 0 & 0 & 0 \\
Neurological & 0 & 0 & 0 & 0 \\
Focal deficits & 1 & 14 & 0 & 0 \\
Delirium/Confusion & & & & \\
Atrial fib & 1 & 14 & 2 & 29 \\
Tamponade/re-exploration & 0 & 0 & 2 & 29 \\
Reperfusion injury/ARDS & 3 & 43 & 4 & 4 \\
Pneumonia & 2 & 29 & 2 & 29 \\
Pneumothorax & 1 & 14 & 0 & 0 \\
Respiratory failure & 0 & 0 & 0 & 0 \\
Pleural effusion & 5 & 71 & 4 & 57 \\
Heparin induced thrombocytopenia & 1 & 14 & 0 & 0 \\
Hemoptysis & 0 & 0 & 1 & 14 \\
Sepsis & 0 & 0 & 1 & 14 \\
Pulmonary thrombosis & 0 & 0 & 0 & 0 \\
Sternal wound infection & 0 & 0 & 1 & 0 \\
\hline
\end{tabular}

hypothermia using the technique of clamping the right innominate and left common carotid arteries. Three groups had previously reported using different versions of antegrade cerebral perfusion for PTE. The Hagl et al., group had performed antegrade brain perfusion at moderate hypothermia $\left(28^{\circ} \mathrm{C}-32^{\circ} \mathrm{C}\right)$, via an aortic inflow cannula. Proximally, an aortic clamp was placed below the inflow cannula and distally they performed balloon occlusion of the descending aorta, near the origin of the bronchial artery [5]. The Thomson et al. group used continuous antegrade cerebral perfusion also via an ascending aortic cannula at $20^{\circ} \mathrm{C}$, with occasional clamping of the ascending aorta, proximally, and the region between the left common carotid and left subclavian artery distally [2]. Masuda et al., used bilateral perfusion of the right axillary and left common carotid artery, with clamping of the ascending aorta, at deep hypothermia $\left(18^{\circ} \mathrm{C}\right)[6]$. The first two groups reported having to resort to DHCA in certain cases to complete the procedure due to significant bronchial artery backflow [2,5]. The third group reported having performed suboptimal dissections in 2 of their 4 cases [6]. We believe that clamping the origins of the left carotid artery and the right innominate artery is the best way to minimize retrograde backflow. Excellent visualization of the endarterectomy plane was obtained in all our cases to perform the meticulous extraction of thro- mbo-embolic material. 
Our study is the first to compare the postoperative hemodynamic parameters between the standard technique to that of selective antegrade brain perfusion with moderate hypothermia. The results obtained suggest that the modified procedure did not negatively affect the postoperative hemodynamic parameters. In fact, there was a significant reduction in postoperative wake up time, and non-significant reduction in duration of surgery, postoperative mechanical ventilation time and need for postoperative inotropic support in the modified group in comparison with the control group.

With a larger sample size and even milder temperatures, we believe that significant differences in duration of surgery can be achieved with the modified technique. Longer duration of surgery implies additional CPB time to cool and re-warm the patient. The prolongation of $\mathrm{CPB}$ can in turn cause increased micro-emboli production and blood component trauma [9].

In our results, we did not find a significant difference, between groups, in postoperative $8 \mathrm{~h}$ blood loss, as collected in the mediastinal sump. Perhaps, a measurement of difference in preoperative to postoperative serum coagulation factors and amount of postoperative blood transfusion requirements, may be a better reflection of hypothermia induced coagulopathy. Inclusion of this data could be relevant for future studies.

A major limitation of our study is the retrospective nature and the small sample size. There may also be a procedural learning bias in comparing the control population, whose surgeries were all performed prior to that of the modified population. However, we attempted to reduce this bias by reporting only the last 7 patients in whom we performed the standard technique; prior to 2008, a total of 21 PTE surgeries had been performed using the standard technique.

A second limitation of our study is the assumption that by performing unilateral perfusion of the right axillary artery, there should be adequate collateral flow between the right and left hemispheres through the circle of Willis (CW). There is sufficient evidence of good cross perfusion of both hemispheres via the $\mathrm{CW}$ in the majority of the population to avoid preoperative CT brain angiography. Firstly, in his study of the anatomy of the CW, Alpers described a normal configuration of the $\mathrm{CW}$ in only $52.3 \%$ of brain speciemens. However, it was noted that a truly incomplete $\mathrm{CW}$, occurred in only $0.005 \%$ of cases [10]. The major anomaly seen was hypoplasia of one or more arteries. Although these hypoplastic vessels may offer increased resistance to collateral flow, histologic studies show that even in their most anomalous form, collateral flow is adequate for nutritional requirement [11]. Secondly, it has been found that anatomic incompleteness of the $\mathrm{CW}$ as determined by $\mathrm{CT}$ angiography does not correlate with actual functional cerebral crossperfusion [12].

\section{Conclusions}

Our results demonstrate that PTE via right axillary antegrade cerebral perfusion at moderate hypothermic temperatures is safe and technically feasible. The technique allows for a bloodless operative field. No permanent or transient neurological complications were seen in the modified group. With a larger population size and even milder cooling, more benefits maybe seen with the modified technique.

\section{References}

[1] S. W. Jamieson, et al., "Pulmonary Endarterectomy: Experience and Lessons Learned in 1500 Cases," The Annals of Thoracic Surgery, Vol. 76, 2003, pp. 1457-1462, 1462-1464.

[2] B. Thomson, et al., "Pulmonary Endarterectomy Is Possible and Effective without the Use of Complete Circulatory Arrest-The UK Experience in Over 150 Patients," European Journal of Cardio-Thoracic Surgery, Vol. 33, No. 2, 2008, pp. 157-163. doi:10.1016/i.ejcts.2007.11.009

[3] J. D. Salazar, et al., "Selective Cerebral Perfusion: RealTime Evidence of Brain Oxygen and Energy Metabolism Preservation," The Annals of Thoracic Surgery, Vol. 88, No. 1, 2009, pp. 162-169. doi:10.1016/j.athoracsur.2009.03.084

[4] C. Staikou, et al., "Impact of Graded Hypothermia on Coagulation and Fibrinolysis," Journal of Surgical Research, 2009. (Epub ahead of print).

[5] C. Hagl, et al., "Technical Advances of Pulmonary Thromboendarterectomy for Chronic Thromboembolic Pulmonary Hypertension," European Journal of CardioThoracic Surgery, Vol. 23, No. 5, 2003, pp. 776-781. doi:10.1016/S1010-7940(03)00029-0

[6] M. Masuda, et al., "Surgical Treatment for Chronic Thromboembolism under Cardiopulmonary Bypass with Selective Cerebral Perfusion," Surgery Today, Vol. 31, No. 2, 2001, pp. 108-112. doi:10.1007/s005950170192

[7] J. F. Sabik, et al., "Axillary Artery: An Alternative Site of Arterial Cannulation for Patients with Extensive Aortic and Peripheral Vascular Disease," Journal of Thoracic and Cardiovascular Surgery, Vol. 109, No. 5, 1995, pp. 885-890, 890-891.

[8] J. F. Sabik, et al., “Axillary Artery Cannulation," The Annals of Thoracic Surgery, Vol. 77, No. 4, 2004, pp. 13151320. doi:10.1016/j.athoracsur.2003.08.056

[9] S. Chanyi, "Cerebral Perfusion and Hypothermic Circulatory Arrest," Journal of Cardiothoracic and Vascular Anesthesia, Vol. 10, No. 1, 1996, pp. 75-82. doi:10.1016/S1053-0770(96)80181-9

[10] B. J. Alpers, et al., "Anatomical Studies of the Circle of Willis in Normal Brain," AMA Archives of Neurology and 
Psychiatry, Vol. 81, No. 4, 1959, pp. 409-418.

[11] H. C. Riggs and C. Rupp, "Variation in Form of Circle of Willis. The Relation of the Variations to Collateral Circulation: Anatomic Analysis," Archives of Neurology, Vol. 8, No. 1, 1963, pp. 8-14.

doi:10.1001/archneur.1963.00460010024002
[12] Urbanski, et al., "Does Anatomical Completeness of the Circle of Willis Correlate with Sufficient Cross-Perfusion during Unilateral Cerebral Perfusion?" European Journal of Cardio-Thoracic Surgery, Vol. 33, No. 3, 2008, pp. 402-408. doi:10.1016/j.ejcts.2007.12.021 